\title{
HF radar for port management: Case study in the port of Rotterdam
}

\author{
M. L. Heron ; A. Prytz ; R. Gomez ; H. Peters
}

The phased array HF ocean radar installed at the Port of Rotterdam gives good quality near real-time information on surface currents for port management. This paper examines the potential to infer current profiles from HF radar and wind station data using prior knowledge of the site. It is shown that tidal currents follow a logarithmic boundary layer profile, and the wind-driven currents have an approximately exponential profile. These two models are combined to estimate the current profiles. 